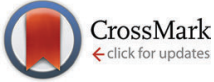

Cite this: J. Mater. Chem. C, 2015, 3, 10616

Received 1st July 2015,

Accepted 1st September 2015

DOI: $10.1039 / c 5 t c 01952 d$

www.rsc.org/MaterialsC

\section{Acido-basic control of the thermoelectric properties of poly(3,4-ethylenedioxythiophene)tosylate (PEDOT-Tos) thin films}

\author{
Zia Ullah Khan, ${ }^{a}$ Olga Bubnova, ${ }^{\text {ab }}$ Mohammad Javad Jafari, ${ }^{c}$ Robert Brooke, ${ }^{\text {ad }}$ \\ Xianjie Liu, ${ }^{C}$ Roger Gabrielsson, ${ }^{a}$ Thomas Ederth, ${ }^{c}$ Drew R. Evans, ${ }^{d}$ \\ Jens W. Andreasen, ${ }^{e}$ Mats Fahlman ${ }^{c}$ and Xavier Crispin ${ }^{a}$
}

\begin{abstract}
PEDOT-TOs is one of the conducting polymers that displays the most promising thermoelectric properties. Until now, it has been utterly difficult to control all the synthesis parameters and the morphology governing the thermoelectric properties. To improve our understanding of this material, we study the variation in the thermoelectric properties by a simple acido-basic treatment. The emphasis of this study is to elucidate the chemical changes induced by acid $(\mathrm{HCl})$ or base $(\mathrm{NaOH})$ treatment in PEDOT-Tos thin films using various spectroscopic and structural techniques. We could identify changes in the nanoscale morphology due to anion exchange between tosylate and $\mathrm{Cl}^{-}$or $\mathrm{OH}^{-}$. But, we identified that changing the $\mathrm{pH}$ leads to a tuning of the oxidation level of the polymer, which can explain the changes in thermoelectric properties. Hence, a simple acid-base treatment allows finding the optimum for the power factor in PEDOT-Tos thin films.
\end{abstract}

\section{Introduction}

Burning fossil fuels is still the main approach to cover the increasing demand of electricity in our society, but the resulting $\mathrm{CO}_{2}$ release is alarming in the context of global warming. Alternative technologies that improve the efficiency of the conversion from fossil energy to electricity are thus highly desirable. Among those, thermoelectric generators constitute a possible emerging technology. For a low temperature region $\left(<250{ }^{\circ} \mathrm{C}\right)$, there exist however no efficient thermoelectric materials that are composed of atomic elements of high natural abundance. This has triggered the research on thermoelectricity towards organic conducting polymers. Indeed, this class of materials could be relatively inexpensive since it is based on atoms of high natural abundance and low toxicity. Moreover conducting polymers are inexpensive to process from solution and do not require any high temperature annealing. ${ }^{1}$

The thermoelectric material efficiency is expressed by the thermoelectric figure-of-merit and given by $Z T=\sigma S^{2} T / \kappa$, where $\sigma$, $S$, and $\kappa$ are the material's electrical conductivity, the Seebeck

\footnotetext{
${ }^{a}$ Department of Science and Technology, Linköping University, Campus Norrköping, S-60174 Norrköping, Sweden.E-mail:xavier.crispin@liu.se

${ }^{b}$ Optoelectronics Group, Cavendish Laboratory, University of Cambridge,

JJ Thomson Avenue, Cambridge CB3 OHE, UK

${ }^{c}$ Department of Physics, Chemistry and Biology, Linköping University,

S-581 83 Linköping, Sweden

${ }^{d}$ University of South Australia, Mawson Institute, Mawson Lakes 5095, Australia

${ }^{e}$ Technical University of Denmark, Department of Energy Conversion and Storage, Frederiksborgvej 399, 4000 Roskilde, Denmark
}

coefficient and thermal conductivity, and $T$ is the operating temperature. One of the most developed and optimized conducting polymers is poly(3,4-ethylenedioxythiophene) displaying very high electrical conductivity up to $4380 \mathrm{~S} \mathrm{~cm}^{-1}$. The thermo- $^{2}$ The electric (TE) efficiency of poly(3,4-ethylenedioxythiophene)tosylate (PEDOT-Tos) has been optimized by chemical doping with the reducing agent tetrakis(dimethylamino)ethylene (TDAE) yielding a maximum $Z T$ of $0.25 .^{3}$ Controlling the oxidation level has also been realized electrochemically., ${ }^{4,5}$ Another recent approach takes the advantage of the known variation of electrical conductivity of the cousin polymer poly(3,4-ethylenedioxythiophene)-poly(styrenesulfonate) (PEDOT-PSS) with $\mathrm{pH}$ to fine tune its thermoelectric properties. ${ }^{6,7}$ However, the power factor achieved with that method $\left(\sigma S^{2}=20 \mu \mathrm{W} \mathrm{K} \mathrm{K}^{-2} \mathrm{~m}^{-1}\right)$ is lower than the record power factor demonstrated for PEDOTPSS ( $470 \mu \mathrm{W} \mathrm{K} \mathrm{K}^{-2} \mathrm{~m}^{-1}, Z T=0.4$ at room temperature) using a post-treatment to remove the excess of insulating PSS. ${ }^{8}$ Recently, we have demonstrated that semi-crystalline PEDOT-Tos is a semimetal composed of a network of bipolarons. The empty bipolaron band overlaps with the filled valence band and this leads to an enhanced Seebeck coefficient. ${ }^{9}$ The electronic structure of PEDOT-Tos is thus totally different than that of polyanilinecamphorsulfonic acid (Pani-CSA), which is metallic ${ }^{10}$ due to the formation of a half-filled polaronic band and possesses a low thermopower. ${ }^{11}$ Electrically conducting polymers are characterized by low thermal conductivity with a clear electronic contribution following the Wiedemann-Franz law. ${ }^{12,13}$ Interestingly, PEDOT-Tos has the same electrical conductivity to thermal conductivity 
ratio as $\mathrm{Bi}_{2} \mathrm{Te}_{3}$ alloys ${ }^{14}\left(\left[600 \mathrm{~S} \mathrm{~cm}^{-1}\right] /\left[1.2 \mathrm{~W} \mathrm{~m}^{-1} \mathrm{~K}^{-1}\right]\right)$, but PEDOT-Tos has a much lower Seebeck effect than its inorganic counterpart. Hence, any strategy that enhances the Seebeck coefficient is of interest.

In this communication, we report the optimization of the thermoelectric properties of PEDOT-Tos films by simply dipping in various $\mathrm{pH}$ solutions. In basic $\mathrm{pH}$, the Seebeck coefficient is enhanced but the electrical conductivity decreases resulting in an optimum $\mathrm{pH}$ region with a maximum power factor. Based on various spectroscopic and structural characterization, we propose a hypothesis to explain the effect of $\mathrm{pH}$ on the thermoelectric properties of PEDOT-Tos.

\section{Experimental}

\subsection{Chemicals}

$40 \mathrm{wt} \%$ solution of iron-tosylate in butanol (Clevios CB 40) was purchased from Heraeus, Germany while the EDOT (3,4-ethylenedioxythiophene) monomer, triblock copolymer poly(ethylene glycol-propylene glycol-ethylene glycol) or PEG-PPG-PEG $\left(M_{\mathrm{w}}=\right.$ $5800 \mathrm{~g} \mathrm{~mol}^{-1}$ ), and pyridine were purchased from Sigma-Aldrich. All these chemicals were used as purchased. A hydrochloric acid (HCL) ACS reagent 37\% was also acquired from Sigma-Aldrich and diluted to get the lower $\mathrm{pH}$ solutions. Sodium hydroxide $(\mathrm{NaOH})$ pellets were purchased from Merck, Germany and dissolved in DI water to get high $\mathrm{pH}$ solutions.

\subsection{PEDOT-Tos film preparation}

PEDOT-Tos films were obtained by vacuum vapor phase polymerization (VVPP) and chemical polymerization (CP).

Higher conductivity PEDOT-Tos samples were prepared using the VVPP technique, where triblock copolymer PEG-PPG-PEG was used to template the growth. The procedure for the VVPP films was similar to those explained previously. ${ }^{15,16}$ Glass substrates were cleaned with ethanol and exposed to an air plasma treatment setup (Diener, Plasma etcher NANO, Germany) for 2 minutes. Oxidant solutions consisting of iron-tosylate (12.3 wt\%) and the triblock copolymer PEG-PPG-PEG (23 wt\%) in butanol were spin coated (400-6NPP, Laurell Technologies Inc.) on the glass substrates for 25 seconds at $1500 \mathrm{rpm}$ immediately after plasma treatment. Samples were then placed on a hot plate at $70{ }^{\circ} \mathrm{C}$ for 30 seconds and then placed immediately into the VVPP chamber with EDOT vapor for a polymerization time of 25 minutes. The chamber temperature was set at $35{ }^{\circ} \mathrm{C}$ with the monomer heated separately to $45{ }^{\circ} \mathrm{C}$. The chamber pressure was set at $45 \mathrm{mbar}$. After polymerization the samples were immediately placed on a hot plate at $70{ }^{\circ} \mathrm{C}$ for 2 minutes and thoroughly washed with ethanol afterwards.

In the case of chemical polymerization (CP), the PEDOT-Tos films were obtained from a mixture of $1 \mathrm{ml}$ of Clevios CB 40 [40 wt\% iron(III)-tosylate in butanol] and $50 \mu \mathrm{l}$ of the EDOT monomer. Furthermore, $0.5 \mathrm{~mol}$ of basic inhibitor pyridine was added per $1 \mathrm{~mol}$ of iron(III)-tosylate so as to increase the $\mathrm{pH}$ of the solution and thereby prevent acid-initiated polymerization that results in poorly conducting films. The solution was spin coated on acetone pre-washed glass substrates at $2000 \mathrm{rpm}$ for
30 seconds. The films were afterwards annealed on a hot plate at $100{ }^{\circ} \mathrm{C}$ for 5 minutes and carefully rinsed in ethanol and deionized (DI) water to remove any unreacted EDOT and residual iron-tosylate and dried by $\mathrm{N}_{2}$. As the DI water has $\mathrm{pH}$ of 5.5 , so all the pristine films are labelled as "pH 5.5 treated" throughout this report.

In both polymerization strategies, the iron-tosylate is used as a reactant to polymerize EDOT but is removed afterwards. During the polymerization, the EDOT radical cations react with each other and form covalent $\mathrm{C}-\mathrm{C}$ bonds between the thiophene units. The dimerization leads to a release of protons. $\mathrm{Fe}^{3+}$ becomes $\mathrm{Fe}^{2+}$ because iron has captured an electron from EDOT. So, for each bond to be formed between two EDOT monomers requires a reaction with iron-tosylate. The PEDOT chains formed are automatically charged positively since it is easier to oxidize an oligomer than a monomer. The positive charge carried by the conducting polymers is balanced by the counter anion tosylate resulting in PEDOT-Tos. After the polymerization, the salt $\mathrm{Fe}^{2+}\left(\mathrm{Tos}^{-}\right)_{2}$ remains in the film which makes the film brown. After a two-step cleaning with ethanol and DI water, the salt goes in the solution and normally there is no iron-tosylate left in the film.

$$
\mathrm{EDOT}+\mathrm{Fe}^{3+}\left(\operatorname{Tos}^{-}\right)_{3} \rightarrow \mathrm{EDOT}^{\bullet+} \operatorname{Tos}^{-}+\mathrm{Fe}^{2+}\left(\operatorname{Tos}^{-}\right)_{2}
$$

To investigate the role of $\mathrm{pH}$ in the thermoelectric performance of PEDOT-Tos, hydrochloric acid $(\mathrm{HCl})$ and sodium hydroxide $(\mathrm{NaOH})$ were used to tune the $\mathrm{pH}$ from 0 to 14 . The films of PEDOT-Tos were dipped in different $\mathrm{pH}$ solutions for two minutes and dried by gentle $\mathrm{N}_{2}$ blowing. The CP PEDOT-Tos samples were produced on plain microscope glass slides for UV-Vis-NIR and GIWAXS, with pre-patterned Au lines on glass for electrical conductivity measurement and with a global coating of $\mathrm{Au}$ on glass for XPS and FTIR measurements.

\subsection{Measurements and characterization}

The resistance $(R)$ of the polymer film was measured with a four probe technique using Keithley 4200 and then the electrical conductivity $(\sigma)$ was calculated by $\sigma=L /(R \cdot w \cdot t)$, where $L, w$ and $t$ were length, width and thickness of the polymer film. The thickness was measured using a Dektak surface profiler and a Scanning Probe Microscope AFM Dimension 3100 from Veeco.

The Seebeck coefficient was measured using a fully automated LabVIEW controlled setup where the sample was placed between two peltier elements with adjustable separation. One of the peltier elements was heated, while the other one was kept at room temperature. The glass slide with a polymer film and calibrated gold thermistors as shown in Fig. 1a was fixed between the hot and cold sides to ensure a good thermal contact within the set-up. The resistances of the two thermistors on the reference sample were measured with the four-point probe method using Keithley 4200, and the temperature difference between two lines was subsequently calculated from the respective calibration slopes. The generated thermoelectric voltage $(\Delta V)$ was measured using a nanovoltmeter Keithley 2182, with one probe each on the hot and cold $\mathrm{Au}$ lines joined by the polymer film in between. The temperature gradient was ramped and the Seebeck coefficient was acquired from the slope of open circuit voltage plotted as a function of temperature difference or $\Delta V / \Delta T$. The temperature was 
(a)

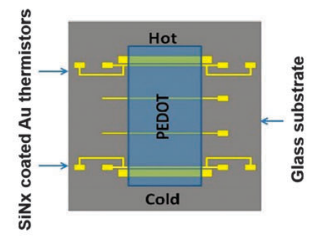

(b)

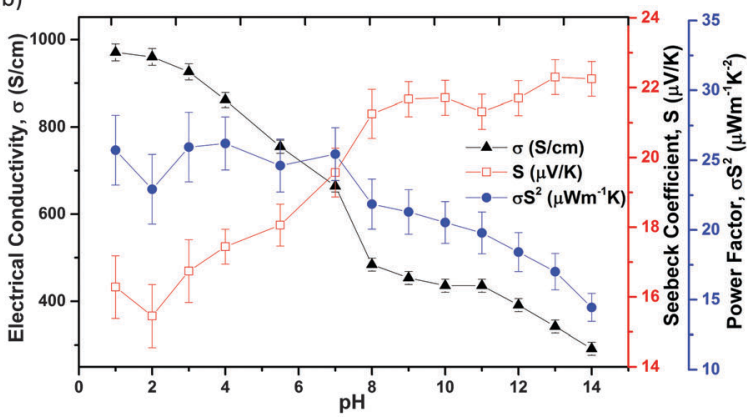

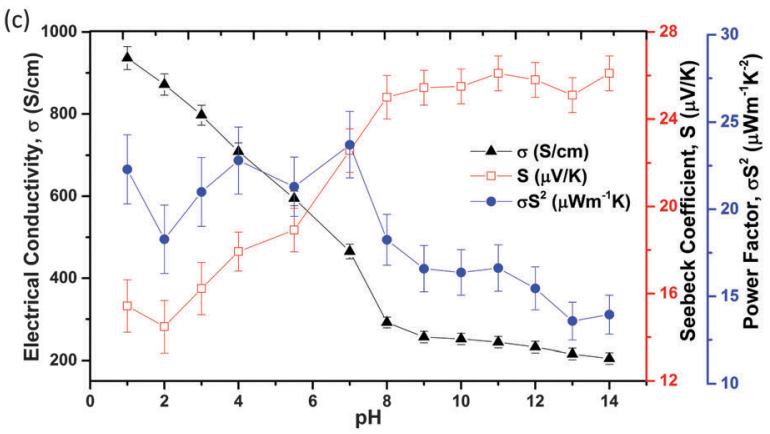

(d)

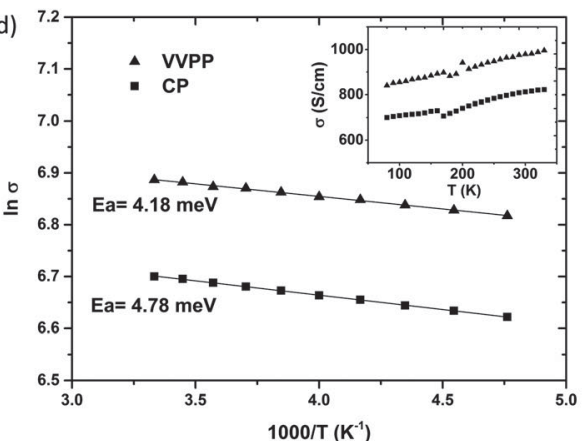

Fig. 1 (a) Schematic of the sample structure for the Seebeck coefficient and electrical conductivity measurement. Glass substrates with patterned Au lines were used as electrodes and thermistors. Thermistors used for temperature measurements were insulated from PEDOT-Tos films with SiN thin layers. (b) TE properties of VVPP PEDOT-Tos films when a single sample is treated with solution of pH 1 to pH 14. (c) TE properties of CP PEDOT-Tos films when one sample is treated with solutions from pH 1 to $\mathrm{pH}$ 14. (d) The Arrhenius plot of pristine (untreated) samples of VVPP and CP PEDOT-Tos in the temperature window of $210-300 \mathrm{~K}$. The inset shows the electrical conductivity of the polymer films as the temperature was decreased from $350 \mathrm{~K}$ to $80 \mathrm{~K}$ in steps of $10 \mathrm{~K}$. Both VVPP and CP films show semi-conducting behavior as the conductivity increases with a rise in temperature.

measured using thermistors and thermocouples. All the values were subsequently corrected by subtracting the absolute Seebeck coefficient $(S)$ of gold. The power factor was calculated by $\sigma S^{2}$.

The temperature dependence was analysed inside Cryogenic Probe Station from Janis under vacuum conditions. The temperature was decreased from $330 \mathrm{~K}$ to $80 \mathrm{~K}$ in steps of $10 \mathrm{~K}$. The four probe resistance was measured using Keithley 4200 and was translated to electrical conductivity using film's geometry.

Grazing Incidence Wide-Angle X-ray Scattering (GIWAXS) experiments were performed using a setup ${ }^{17}$ based on a rotating $\mathrm{Cu}$-anode as an X-ray source with a 1D multilayer optic yielding focused and monochromatic X-ray $\mathrm{Cu} \mathrm{K} \alpha$ radiation (wavelength $\lambda=1.5418 \AA$ A). The incidence angle used was $0.18^{\circ}$, just below the critical angle for the glass substrates. The measurements were done in a vacuum, at room temperature. The scattered radiation was collected using a photostimulable imaging plate placed at $119 \mathrm{~mm}$ from the sample. The GIWAXS patterns are represented in reciprocal space coordinates with the scattering vector $Q_{x y}$ in the substrate plane and $Q_{z}$ along the surface normal. The magnitude of the scattering vector $Q$ is defined as $Q=4 \times \mathrm{pi} / \sin \theta$, where $2 \theta$ is the total scattering angle.

Photoemission experiments were carried out using a Scienta ESCA 200 spectrometer in a ultrahigh vacuum with a base pressure of $10^{-10} \mathrm{mbar}$. The measurement chamber is equipped with a monochromatic $\mathrm{Al}(\mathrm{K} \alpha) \mathrm{X}$-ray source providing photons with $1486.6 \mathrm{eV}$ for XPS (X-ray photoemission spectroscopy). The XPS experimental condition was set so that the full width at half maximum of the clean $\mathrm{Au} 4 \mathrm{f}^{7 / 2}$ line was $0.65 \mathrm{eV}$. All spectra were collected at room temperature with a photoelectron take off angle of $0^{\circ}$ (normal emission).

Absorption spectra were studied using a UV-Vis-NIR Spectrometer PerkinElmer Lambda 900 in the range of $1400-300 \mathrm{~nm}$ and the spectra were normalized according to the thickness of the film.

Fourier transform infra-red absorption spectroscopy measurements were carried out by means of a grazing angle $\left(85^{\circ}\right)$ reflection setup in a Bruker IFS66 spectrometer with a $\mathrm{LN}_{2}$-cooled MCT detector. The system was continuously purged with nitrogen before and during the measurement. All spectra were acquired at $2 \mathrm{~cm}^{-1}$ resolution between 4000 and $600 \mathrm{~cm}^{-1}$ and background spectra were recorded using gold coated substrates.

It is worth mentioning that thermoelectric (TE) properties were measured for one sample each of VVPP and CP PEDOT-Tos. The absorption spectroscopy was carried out on one CP film. For the later characterization, distinct CP samples were prepared for every $\mathrm{pH}$ treatment; 8, 7 and 12 samples were used for XPS, GIWAXS and FTIR spectroscopy.

\section{Results}

\subsection{Thermoelectric (TE) properties}

The pristine (untreated) VVPP film had an electrical conductivity of $818 \mathrm{~S} \mathrm{~cm}^{-1}$ with a Seebeck coefficient of $\sim 17 \mu \mathrm{V} \mathrm{K}{ }^{-1}$ while the $\mathrm{CP}$ film resulted in an electrical conductivity of 
$810 \mathrm{~S} \mathrm{~cm}^{-1}$ and a Seebeck coefficient of $16 \mu \mathrm{V} \mathrm{K}^{-1}$. The VVPP PEDOT-Tos film was treated with $\mathrm{pH} 1$ till $\mathrm{pH} 14$ solutions and the thermoelectric properties of this single sample were tracked as shown in Fig. 1b. The electrical conductivity $(\sigma)$ increased to $\sim 970 \mathrm{~S} \mathrm{~cm}^{-1}$ after $\mathrm{pH} 1$ treatment and decreased down to $290 \mathrm{~S} \mathrm{~cm}^{-1}$ at pH 14 treatment while the Seebeck coefficient $(S)$ increased from 15 to $23 \mu \mathrm{V} \mathrm{K} \mathrm{K}^{-1}$. The power factor $\sigma S^{2}$ was around $26 \mu \mathrm{W} \mathrm{K} \mathrm{K}^{-2} \mathrm{~m}^{-1}$ for $\mathrm{pH} \leq 7$ treated films and it decreased with base treatment because of the contradicting trends of $\sigma$ and $S$.

The chemically polymerized (CP) films were also treated with pH 1-14, to investigate the effects as shown in Fig. 1c. The electrical conductivity was $936 \mathrm{~S} \mathrm{~cm}^{-1}$ at $\mathrm{pH} 1$ treatment which decreased to $204 \mathrm{~S} \mathrm{~cm}^{-1}$ at pH 14 treatment. The corresponding
Seebeck coefficient was at its lowest of $15 \mu \mathrm{V} \mathrm{K}{ }^{-1}$ at pH 1 which increased to $26 \mu \mathrm{V} \mathrm{K}^{-1}$ at $\mathrm{pH} 14$ treatment. Again we see the highest power factor $\sigma S^{2}$ of $23 \mu \mathrm{W} \mathrm{K} \mathrm{K}^{-2} \mathrm{~m}^{-1}$ for $\mathrm{pH} \leq 7$ treated films which decreased after base treatments.

So, it can be seen that both types of PEDOT-Tos films undergo similar changes in thermoelectrical properties after acid/base treatments: from $\mathrm{pH} 1$ to 8 the electrical conductivity reduces sharply (from 970 to $485 \mathrm{~S} \mathrm{~cm}^{-1}$ for VVPP and from 935 to $292 \mathrm{~S} \mathrm{~cm}^{-1}$ for CP films) compared to the changes in the basic treatments from $\mathrm{pH} 8$ to 14 (from 485 to $290 \mathrm{~S} \mathrm{~cm}^{-1}$ for VVPP and 292 to $205 \mathrm{~S} \mathrm{~cm}^{-1}$ for the CP film). The fact that a similar trend is observed with and without the introduction of the copolymer PEG-PPG-PEG indicates that the latter has no (a)

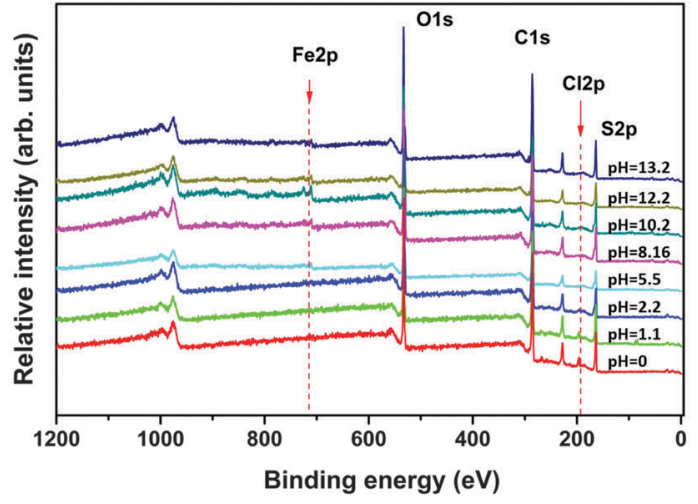

(c)

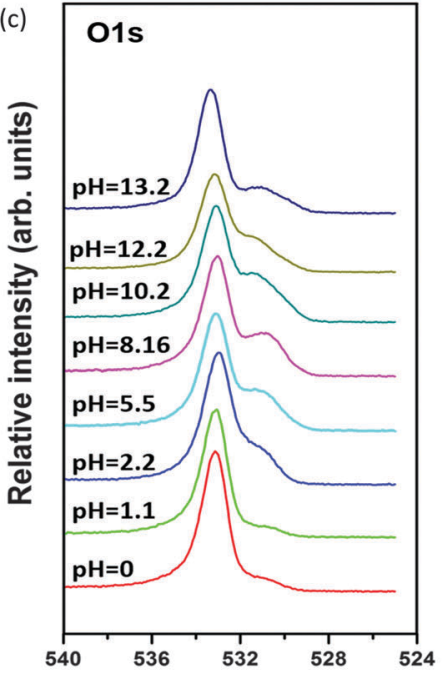

Binding energy (eV) (b)

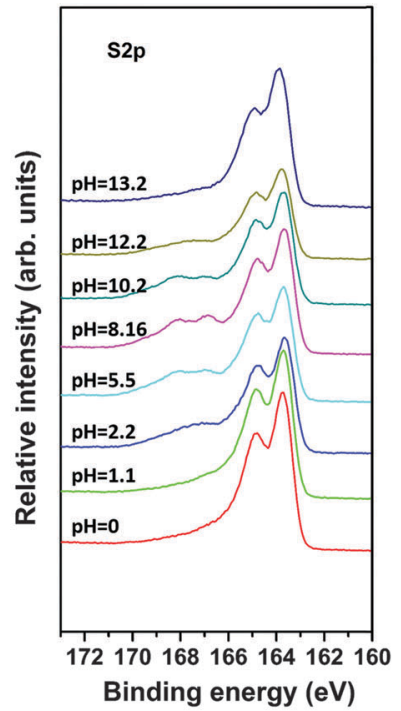

(d)

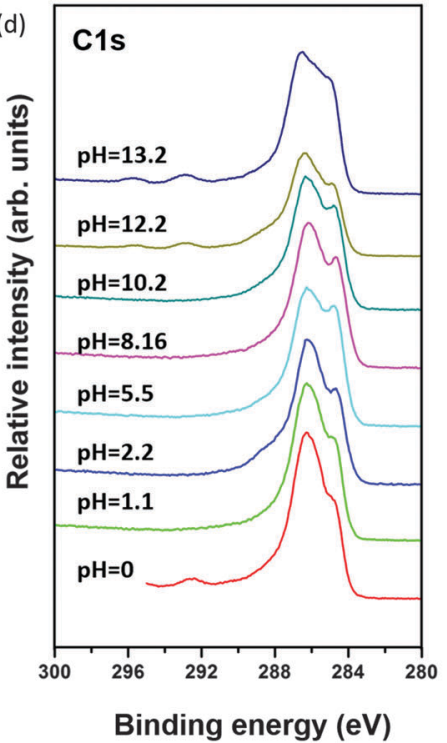

Fig. 2 XPS spectra where distinct CP PEDOT-Tos samples were treated with different pH solutions. The pristine (untreated) sample is labelled as pH 5.5 in all four spectra (a) complete wide-scan XPS spectra showing the presence of $\mathrm{Cl}^{-}$in lower $\mathrm{pH}$ treated samples (b) S2p spectra showing a diminishing amount of tosylate as the film is treated with low or high $\mathrm{pH}$ solutions (c) O1s spectra showing the presence of an oxygen peak at 531 eV for high $\mathrm{pH}$ treatment when $\mathrm{OH}^{-}$is present while it reduces at low $\mathrm{pH}$ when $\mathrm{Cl}^{-}$is replacing tosylate (d) $\mathrm{C} 1 \mathrm{~s}$ spectra showing new peaks at $\mathrm{pH}>10$ due to the presence of new $\mathrm{C}=\mathrm{O}$ bonds indicating ring opening. 
effect on the $\mathrm{pH}$ dependence. Based on that observation, we characterize the chemical changes taking place in the CP films, i.e. without copolymers, in order to avoid the additional complexity in the interpretation of the XPS, GIWAXS and FTIR data that would come with the copolymer.

The temperature dependencies of the pristine (untreated) VVPP and CP films were analysed in the range of $80-330 \mathrm{~K}$ as shown in Fig. 1d. It is clear from these plots that both of these polymer films were semiconducting in behaviour. The activation energies of $4.18 \mathrm{meV}$ for the VVPP and $4.78 \mathrm{meV}$ for the CP films were calculated from the Arrhenius plots as shown in Fig. 1d in the temperature window of 210-300 K. Note that even if the composition of the PEDOT-Tos films is similar to the one reported with semimetallic behaviour, ${ }^{9}$ its electrical behaviour is different possibly due to a different morphology.

Although such a phenomenon has been reported for other derivatives of PEDOT, ${ }^{6,7,18}$ it was important to understand the variation in thermoelectric properties of PEDOT-Tos after acid/ base treatments. There can be two possible reasons for these changes: either a change in the morphology and packing of the polymer film and/or a change in the oxidation level. The chemically polymerized (CP) PEDOT-Tos films were selected for such investigations as they do not contain the PEG-PPG-PEG and can be analysed with lesser complexity even if exposed to higher concentrations of $\mathrm{HCl}$ and $\mathrm{NaOH}$.

\subsection{Elemental composition and morphology}

In order to track modification in the chemical composition of the polymer films, we have used X-ray photoelectron spectroscopy (XPS). The spectral shape of C(1s), O(1s), and S(2p) was changed significantly for samples exposed to different $\mathrm{pH}$ solutions compared with the pristine ( $\mathrm{pH} 5.5$ ) sample. Fig. $2 \mathrm{~b}$ displays the two $\mathrm{S}(2 \mathrm{p})$ doublet signals. The $\mathrm{S}(2 \mathrm{p})$ photoelectrons from the sulfonate group of Tos (166-170 eV) have higher binding energy than that coming from the thiophene units in PEDOT (163-166 eV) because the sulfur atoms are bound to three electronegative oxygen atoms. ${ }^{19}$ By following the evolution of the $\mathrm{S}(2 \mathrm{p})$ doublet of Tos, we clearly see that the Tos content diminishes when going to acidic or basic conditions. This indicates that $\mathrm{Tos}^{-}$is replaced by $\mathrm{Cl}^{-}$or $\mathrm{OH}^{-}$through an ion exchange process driven by the chemical potential equalization. For the acidic treatments, the presence of $\mathrm{Cl}^{-}$in the film is visible in a wide-scan XPS (see Fig. 2a). After basic treatment, the Tos anions are exchanged with hydroxyl $\mathrm{OH}^{-}$anions transforming PEDOT-Tos into PEDOT-OH as suggested by the presence of an $\mathrm{O}(1 \mathrm{~s})$ singlet at $531 \mathrm{eV}$ more pronounced at high $\mathrm{pH}$ than low $\mathrm{pH}$, but maximum at neutral $\mathrm{pH}$ when Tos is the counterion (with 3 oxygen atoms per anion) as shown in Fig. 2c.

At $\mathrm{pH}>10$, one can see two new peaks in the C1s spectra as shown in Fig. 2d at very high binding energy (292 eV, $295 \mathrm{eV})$. So, it typically represents a carbon attached to 2 oxygen atoms, like in carboxylic or ester groups. The existence of this $\mathrm{C}=\mathrm{O}$ type stretching at high $\mathrm{pH}$ may also indicate ring opening damaging the sigma bonds in the PEDOT backbone.

In order to investigate how the ion exchange influences the morphology and molecular ordering, we systematically follow the diffraction patterns obtained by Grazing Incidence Wide Angle X-ray Scattering (GIWAXS) versus $\mathrm{pH}$. GIWAXS data were acquired for pristine films as well as films treated with solutions of $10 \mathrm{M}, 1 \mathrm{M}$ and $0.01 \mathrm{M} \mathrm{HCl}$ and $\mathrm{NaOH}$. The pristine film and
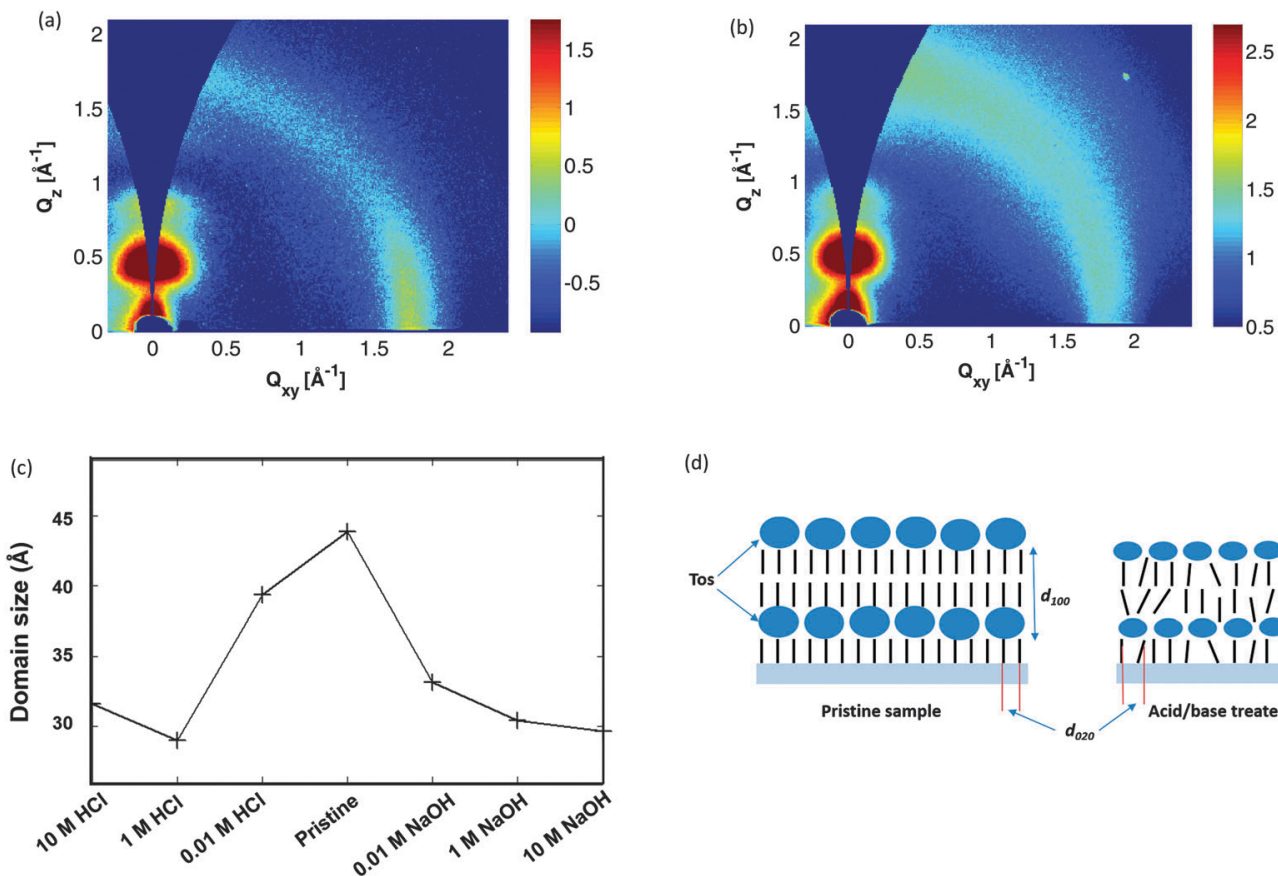

(d)

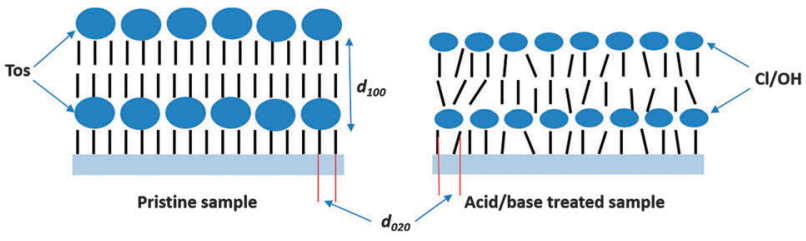

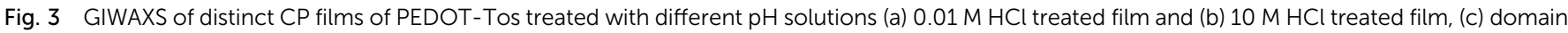

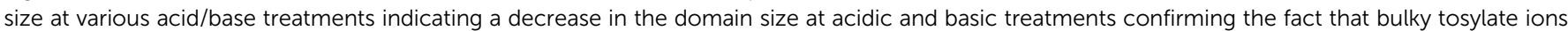

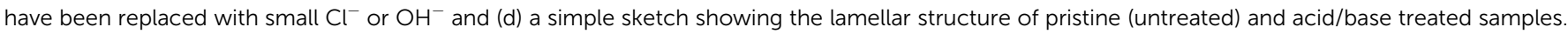


the films treated with weak acid or base show the typical texture of a spin coated film of a semi-crystalline conjugated polymer, with "edge-on" orientation, i.e. with the aromatic planes of the backbone oriented perpendicular to the substrate surface and the $\pi$-stacking along the surface (Fig. 3a). At higher acid or base concentration, the diffracted intensity at the scattering vector corresponding to the $\pi$-stacking inter-chain distance $(\sim 3.5 \AA)$ is more evenly distributed over azimuthal angles, whereas the azimuthal distribution of intensity in the lamellar peak is unaffected (Fig. 3b). The latter observation indicates that the effect of acid or base treatment is not an overall change in the domain texture, but rather a disruption of the $\pi$-stacking alone, with ordering along the surface normal left more or less unaffected. A closer examination of the data reveals however that the domain size along the surface normal is diminished by about $30 \%$ in films treated with strong acid or base as shown in Fig. 3c. The effect has been shown with a simple sketch in Fig. 3d where bulky tosylate has been replaced with atomic counterions which affects the lamellar spacing (indicated as $d_{100}$ in Fig. 3d) and the $\pi$-stacking (indicated by $d_{020}$ in Fig. $3 \mathrm{~d}$ ).

From the XPS and GIWAXS analysis, we understand that the acido-basic treatment leads to an ion-exchange that actually disrupts the overlap between the $\pi$-orbitals of adjacent PEDOT chains; which is thus expected to deteriorate the charge transport. But systematically, the inter-stack distance decreases when Tos is replaced by smaller anions; which could favour inter-stack transport. But none of those observations can explain the experimental observation of the electrical conductivity versus $\mathrm{pH}$; which displays an opposite trend for the acid treatment. This motivates us to investigate further if the electronic structure of PEDOT is changed versus $\mathrm{pH}$.

\subsection{Electronic and geometrical structure changes}

In order to probe the $\pi$-electronic system of PEDOT, we follow the optical absorption spectrum in the UV-Vis-NIR range as shown in Fig. 4a as well as the vibrational spectra with Fourier transform infra-red (FTIR) spectroscopy as shown in Fig. 5a. Up to $\mathrm{pH}=3$, a slight reduction of the NIR background is observed in both the optical and FTIR spectra and associated with a slight reduction of the free charge carriers, possibly as a result of a localization of some bipolaron wavefunction upon ion exchange. From $\mathrm{pH}=3$ to 8 , the IR background continues to decrease and two polaronic transitions appear more pronounced at $900 \mathrm{~nm}^{20}$ and above $1400 \mathrm{~nm}^{21}$ while only a minor increase in absorption is observed in the visible region as shown in Fig. 4a. Those observations suggest that going towards basic solution, PEDOT undergoes a reduction. The electron transfer to PEDOT transforms bipolarons into polarons. Similar optical features accompanied by the presence of unpaired electrons, i.e. polaronic defects, are observed in several studies with EPR upon decrease of the oxidation level in PEDOT. ${ }^{22}$ Higher $\mathrm{pH}$ values induce an increased absorption in the visible range (peak at $600 \mathrm{~nm}$ ) indicating the occurrence of neutral species on the polymer chains as shown in Fig. $4 \mathrm{a}$ and $\mathrm{b}$. The nonlinear evolution of the absorption at $600 \mathrm{~nm}$ versus $\mathrm{pH}$ indicates that the reduction takes place in two steps (Fig. $4 \mathrm{~b}$ ): from the bipolaron to polaron, then from the polaron to neutral segment.
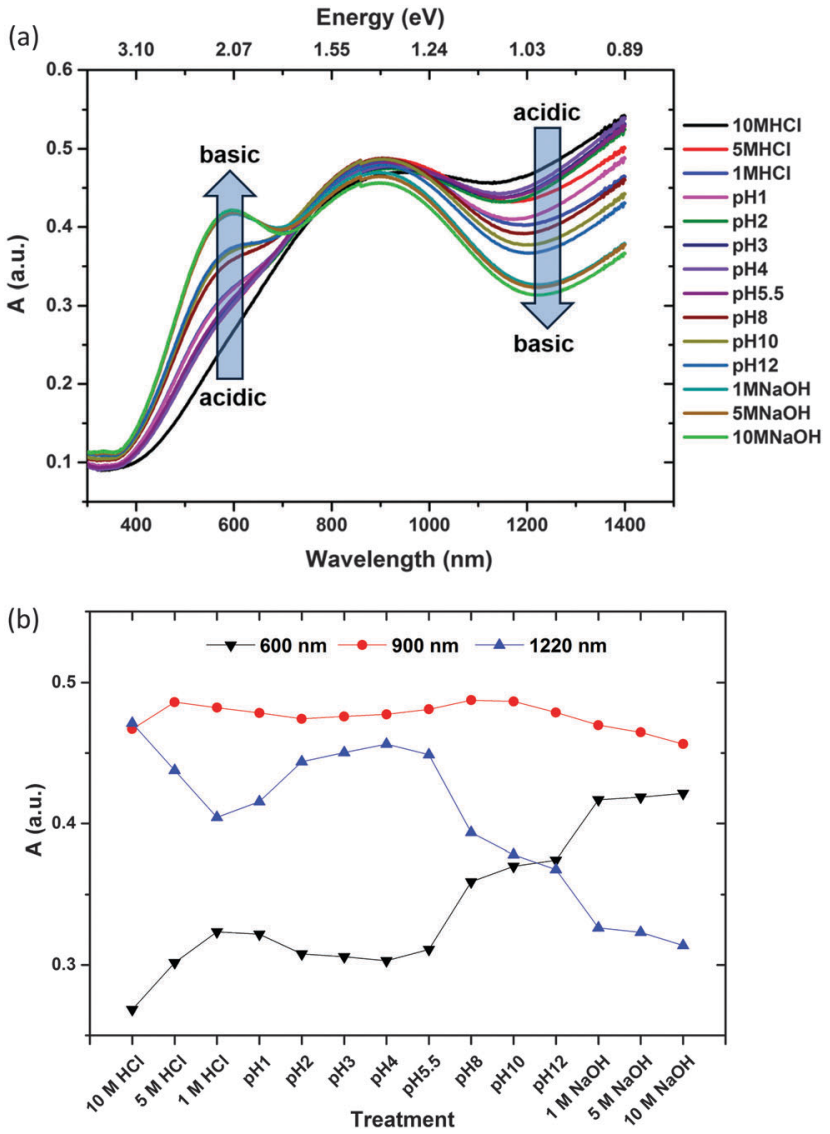

Fig. 4 (a) Absorption spectra of one CP PEDOT-Tos film treated from $10 \mathrm{M}$ $\mathrm{HCl}$ to $10 \mathrm{M} \mathrm{NaOH}$. The arrow indicates the trends while going from acidic to basic treatments of the film, (b) absorption at 600,900 and $1220 \mathrm{~nm}$ after acid/ base treatment. While going from acidic towards basic treatments, there is a decrease in absorption at $\sim 1220 \mathrm{~nm}$ and an enhancement in the peak at $\sim 600 \mathrm{~nm}$, indicating quenching of bipolarons and appearance of neutral segments in PEDOT-Tos films causing a decrease in the electrical conductivity.

The FTIR spectra of PEDOT-Tos samples treated with different $\mathrm{pH}$ in the wavenumber region $1800-800 \mathrm{~cm}^{-1}$ is shown in Fig. $5 \mathrm{a}$. For the pristine ( $\mathrm{pH}$ 5.5) sample, the IRAS spectrum shows a number of peaks assigned to PEDOT and tosylate units. The band at 1529 (with a shoulder at $1476 \mathrm{~cm}^{-1}$ ) is due to asymmetric $\mathrm{C}_{\alpha}=\mathrm{C}_{\beta}$ stretching in the thiophene ring ${ }^{23}$ and the symmetric $\mathrm{C}_{\alpha}=\mathrm{C}_{\beta}(-\mathrm{O})$ stretch vibration is found at $1418 \mathrm{~cm}^{-1}$. $^{23}$ The peak at $1372 \mathrm{~cm}^{-1}$ and the broad and weak band centered at $1308 \mathrm{~cm}^{-1}$ are also attributed to $\mathrm{C}_{\beta}-\mathrm{C}_{\beta}$ and $\mathrm{C}_{\alpha}-\mathrm{C}_{\alpha^{\prime}}$ inter-ring stretch, respectively. ${ }^{23,24}$ The vibrations at 1200, 1106, 1072 and $923 \mathrm{~cm}^{-1}$ are attributed to $\mathrm{C}-\mathrm{O}-\mathrm{C}$ stretching modes and ring vibrations and deformation characteristics of the ethylenedioxy group. ${ }^{24}$ The bands observed at 977 and $845 \mathrm{~cm}^{-1}$ are assigned to stretching and deformation of the $\mathrm{C}-\mathrm{S}-\mathrm{C}$ bond in the thiophene ring. ${ }^{25}$ Several weak bands and shoulders corresponding to the tosylate unit appear in the spectrum, which can be identified as asymmetric $\left(1274 \mathrm{~cm}^{-1}\right)$ and symmetric $\left(1143 \mathrm{~cm}^{-1}\right) \mathrm{S}=\mathrm{O}$ and S-phenyl $\left(1011 \mathrm{~cm}^{-1}\right)$ vibrations. ${ }^{23}$ The low intensities of these bands are related to the low concentration of tosylates in the sample.

There is a higher amount of tosylate in the pristine sample (treated with $\mathrm{pH}$ 5.5) but at high $\mathrm{pH}(\mathrm{pH}>10)$ these peaks 

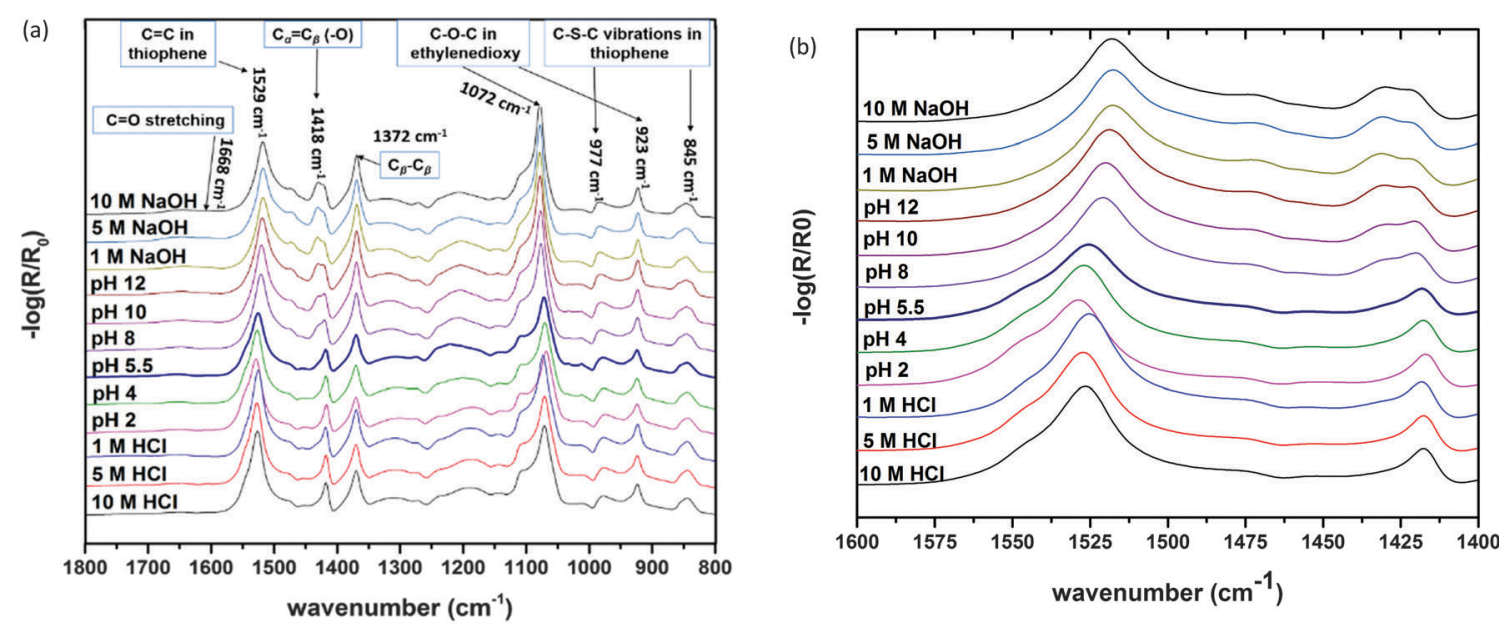

(c)
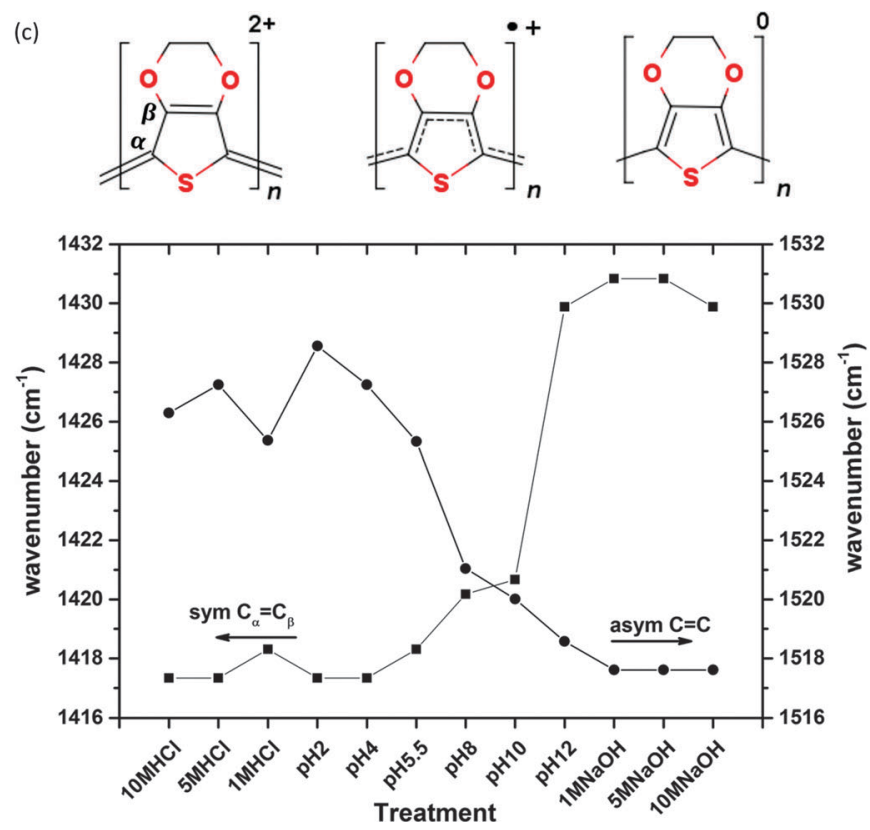

Fig. 5 The FTIR spectra of distinct CP films of PEDOT-Tos treated with acid/base solutions. The pristine (untreated) film is labelled as pH 5.5 treated (a) full FTIR spectra showing the finger print regions of PEDOT (b) FTIR spectra at $1600-1400 \mathrm{~cm}^{-1}$ showing the shift of $\mathrm{C}=\mathrm{C}$ stretch to lower wavenumbers while the $C_{\alpha}=C_{\beta}$ stretch shifts to higher wavenumbers affecting the bonding pattern (c) peak position variation indicating the transformation of the quinoid into benzoid structure as we go from acidic to basic treatment of the polymer films resulting in a decrease in electrical conductivity.

disappear showing the removal of tosylate from the film. The position of $\mathrm{C}_{\alpha}=\mathrm{C}_{\beta}$ peak shifts from 1418 to $1430 \mathrm{~cm}^{-1}$ at $\mathrm{pH}>$ 10 as shown in Fig. 5b and $\mathrm{c}$ with a new peak also appearing at $1433 \mathrm{~cm}^{-1}$ which can be attributed to transformation from the quinoid to benzoid structure of PEDOT. Positive (bi)polarons are positively charged doped species that are located on a geometrical defect. The geometrical defect is typically quinoid in nature. Intensity of a new peak $\left(1433 \mathrm{~cm}^{-1}\right)$ increases for higher $\mathrm{pH}$ and can show that more and more quinoids are transformed into benzoid with an increase of $\mathrm{pH}$, indicating a decrease in the amount of (bi)polarons subsequent to a reduction of PEDOT. Fig. $5 \mathrm{c}$ shows a change in the position of the $\mathrm{C}=\mathrm{C}$ peak from $1526 \mathrm{~cm}^{-1}$ at lower $\mathrm{pH}$ to $1518 \mathrm{~cm}^{-1}$ at higher $\mathrm{pH}$ indicating structural transformation of the PEDOT unit at high $\mathrm{pH}$. At $\mathrm{pH}>10$, a broad peak (centered at $1668 \mathrm{~cm}^{-1}$ as shown in Fig. 5a) also appeared and its intensity increases with an increase of $\mathrm{pH}$ which can be assigned to the carbonyl group and $\mathrm{C}=\mathrm{O}$ stretching. This is consistent with the degradation mechanism proposed from the XPS C(1s) peak observed at high binding energy. From the FTIR spectroscopy, it can be concluded that the decrease in electrical conductivity (from acidic to basic treatments) can be attributed to structural transformation (quinoid to benzoid) ${ }^{26}$ and ring opening along the PEDOT chain.

\section{Discussion}

Besides the irreversible degradation mechanism at $\mathrm{pH}>10$, the evolution of the Seebeck coefficient and electrical conductivity with $\mathrm{pH}$ is reversible and is primarily due to a change in 
the oxidation level of the PEDOT and to a less extent a change in morphology. It has indeed been reported that the electrical conductivity decreases and the Seebeck coefficient increases when the oxidation level (doping) is reduced; ${ }^{3,5}$ which is fully consistent with our finding. One hypothesis that can explain the $\mathrm{pH}$ effect is a protonation of the end-monomer in a PEDOT chain. Indeed, a protonation would be equivalent to introducing a positive charge in the $\pi$-system, i.e. equivalent to an oxidation. This effect can be significant because in PEDOT-Tos, the PEDOT chains have most likely a low molecular weight.

\section{Conclusions}

Thermoelectric properties (electrical conductivity and Seebeck coefficient) of the high conductivity VVPP films and CP PEDOTTos films were studied while exposing these films to different $\mathrm{pH}$ solutions ( $\mathrm{pH} 1$ to $\mathrm{pH}$ 14). The XPS and GIWAXS demonstrate that the Tosylate ions are replaced by $\mathrm{Cl}^{-}$or $\mathrm{OH}^{-}$anions; which subsequently affects the packing of the PEDOT chains in the film. However, this cannot explain the observed trends for the thermoelectric properties with $\mathrm{pH}$ : the electrical conductivity falls and the thermopower is enhanced as the film is treated with more basic solutions. This has been attributed to a change in the oxidation level as indicated by a clear modification of the optical absorption revealing neutral PEDOT segments at basic $\mathrm{pH}$ : a transition from the bipolaronic to polaronic and the polaronic to neutral state as seen by the increased absorption in the visible region in IR spectra. The IRAS spectra also support this modification in the oxidation level since the bonding pattern changes from the quinoid to benzoid structure. We get the highest power factor $\left(\sigma S^{2}\right)$ of $26 \mu \mathrm{W} \mathrm{K} \mathrm{K}^{-2} \mathrm{~m}^{-1}$ at $\mathrm{pH} \leq 7$.

\section{Acknowledgements}

The authors acknowledge the European Research Council (ERC-starting-grant 307596), the Swedish foundation for strategic research (project: "Nano-material and Scalable TE materials"), the Knut and Alice Wallenberg foundation (project "Power Paper" and "Tail of the Sun"), The Swedish Energy Agency and the Advanced Functional Materials Center at Linköping University.

\section{References}

1 O. Bubnova and X. Crispin, Energy Environ. Sci., 2012, 5, 9345-9362.

2 N. Kim, S. Kee, S. H. Lee, B. H. Lee, Y. H. Kahng, Y. R. Jo, B. J. Kim and K. Lee, Adv. Mater., 2014, 26, 2268-2272.

3 O. Bubnova, Z. U. Khan, A. Malti, S. Braun, M. Fahlman, M. Berggren and X. Crispin, Nat. Mater., 2011, 10, 429-433.

4 T. Park, C. Park, B. Kim, H. Shin and E. Kim, Energy Environ. Sci., 2013, 6, 788-792.

5 O. Bubnova, M. Berggren and X. Crispin, J. Am. Chem. Soc., 2012, 134, 16456-16459.
6 F. Kong, C. Liu, F. Jiang, J. Xu, B. Lu, R. Yue and S. Chen, Presented in part at the 2010 International Conference on Digital Manufacturing and Automation (ICDMA), Changsha, China, 2010, pp. 655-658.

7 T.-C. Tsai, H.-C. Chang, C.-H. Chen, Y.-C. Huang and W.-T. Whang, Org. Electron., 2014, 15, 641-645.

8 G. H. Kim, L. Shao, K. Zhang and K. P. Pipe, Nat. Mater., 2013, 12, 719-723.

9 O. Bubnova, Z. U. Khan, H. Wang, S. Braun, D. R. Evans, M. Fabretto, P. Hojati-Talemi, D. Dagnelund, J.-B. Arlin, Y. H. Geerts, S. Desbief, D. W. Breiby, J. W. Andreasen, R. Lazzaroni, W. M. Chen, I. Zozoulenko, M. Fahlman, P. J. Murphy, M. Berggren and X. Crispin, Nat. Mater., 2014, 13, 190-194.

10 K. Lee, S. Cho, S. Heum Park, A. J. Heeger, C.-W. Lee and S.-H. Lee, Nature, 2006, 441, 65-68.

11 S. Stafström, J. L. Brédas, A. J. Epstein, H. S. Woo, D. B. Tanner, W. S. Huang and A. G. MacDiarmid, Phys. Rev. Lett., 1987, 59, 1464-1467.

12 A. Weathers, Z. U. Khan, R. Brooke, D. Evans, M. T. Pettes, J. W. Andreasen, X. Crispin and L. Shi, Adv. Mater., 2015, 27, 2101-2106.

13 J. Liu, X. Wang, D. Li, N. E. Coates, R. A. Segalman and D. G. Cahill, Macromolecules, 2015, 48, 585-591.

14 S. Scherrer and H. Scherrer, CRC Handbook of Thermoelectrics, CRC Press, 1995, DOI: 10.1201/9781420049718.ch19.

15 D. Evans, M. Fabretto, M. Mueller, K. Zuber, R. Short and P. Murphy, J. Mater. Chem., 2012, 22, 14889-14895.

16 M. Fabretto, C. Jariego-Moncunill, J.-P. Autere, A. Michelmore, R. D. Short and P. Murphy, Polymer, 2011, 52, 1725-1730.

17 D. Apitz, R. P. Bertram, N. Benter, W. Hieringer, J. W. Andreasen, M. M. Nielsen, P. M. Johansen and K. Buse, Phys. Rev. E: Stat., Nonlinear, Soft Matter Phys., 2005, 72, 036610.

18 Y. J. Xia and J. Y. Ouyang, ACS Appl. Mater. Interfaces, 2010, 2, 474-483.

19 X. Crispin, S. Marciniak, W. Osikowicz, G. Zotti, A. van der Gon, F. Louwet, M. Fahlman, L. Groenendaal, F. De Schryver and W. R. Salaneck, J. Polym. Sci., Part B: Polym. Phys., 2003, 41, 2561-2583.

20 M. Dietrich, J. Heinze, G. Heywang and F. Jonas, J. Electroanal. Chem., 1994, 369, 87-92.

21 K. Jeuris, L. Groenendaal, H. Verheyen, F. Louwet and F. De Schryver, Synth. Met., 2003, 132, 289-295.

22 A. Zykwinska, W. Domagala, A. Czardybon, B. Pilawa and M. Lapkowski, Chem. Phys., 2003, 292, 31-45.

23 F. Ely, A. Matsumoto, B. Zoetebier, V. S. Peressinotto, M. K. Hirata, D. A. de Sousa and R. Maciel, Org. Electron., 2014, 15, 1062-1070.

24 S. Garreau, G. Louarn, J. P. Buisson, G. Froyer and S. Lefrant, Macromolecules, 1999, 32, 6807-6812.

25 S. Nagarajan, J. Kumar, F. F. Bruno, L. A. Samuelson and R. Nagarajan, Macromolecules, 2008, 41, 3049-3052.

26 J. Ouyang, Q. Xu, C.-W. Chu, Y. Yang, G. Li and J. Shinar, Polymer, 2004, 45, 8443-8450. 